\title{
EL PAPEL DE LA SEGUNDA LENGUA EXTRANJERA EN LOS ESTUDIOS DE TRADUCCIÓN EN ESPAÑA: ALGUNAS REFLEXIONES DESDE EL ENFOQUE CULTURAL ${ }^{1}$
}

\author{
Richard Clouet \\ Manuel Wood Wood
}

\begin{abstract}
RESUMEN
El estudio de la segunda lengua extranjera para traductores (Lengua C) debería dar mayor importancia a la dimensión sociocultural y, sobre todo, a la perspectiva intercultural. En el presente trabajo, ofrecemos algunas reflexiones sobre la idea de que la competencia sociocultural es tan importante en la enseñanza de una segunda lengua extranjera como la lengua en sí misma, porque lengua y cultura son dos caras de una misma realidad. La lengua es el vivo y siempre cambiante reflejo de la historia y el saber del pueblo que la utiliza, y en ella cristaliza su experiencia cultural $\mathrm{y}$ archiva todas sus vivencias.
\end{abstract}

Palabras clave: cultura, didáctica de lenguas, enseñanza, traducción.

\begin{abstract}
The study of a second foreign language in Translation Faculties in Spain should give more importance to the socio-cultural dimension of language teaching/learning and especially to the intercultural perspective. This paper offers some reflections on the idea that the socio-cultural competence is as important in teaching a second foreign language as in the mother tongue, since language and culture are undividable realities. Language is the true and ever changing reflection of the history and knowledge of the people that use it and it is precisely where their cultural experience and memories remain crystallised.

Key words: culture, didactics of languages, teaching, translation.
\end{abstract}

Richard Clouet. Doctor en Filología Inglesa. Profesor de Lengua Inglesa en la Facultad de Traducción e Interpretación, Universidad de Las Palmas de Gran Canaria.

Correo electrónico: rclouet@dfm.ulpgc.es

Manuel Wood Wood. Doctor en Filología Inglesa. Profesor de las Facultades de Traducción e Interpretación y Formación del Profesorado, Universidad de Las Palmas de Gran Canaria.

Correo electrónico: mwood@dfm.ulpgc.es

Recepción: 4- 3- 2008

Aceptación: 7- 4- 2008 


\section{El papel de las lenguas extranjeras en la Unión Europea}

Con la incorporación el 1 de enero de 2007 de dos nuevos países de Europa Oriental, Bulgaria y Rumania, la Unión Europea cuenta con veintisiete Estados miembros. Desde entonces, las instituciones europeas reconocen veintitrés lenguas oficiales: el alemán, el búlgaro, el checo, el danés, el eslovaco, el esloveno, el español, el estonio, el finlandés, el francés, el griego, el húngaro, el inglés, el irlandés, el italiano, el letón, el lituano, el maltés, el neerlandés, el polaco, el portugués, el rumano y el sueco. Además de estas veintitrés lenguas oficiales, los ciudadanos y representantes políticos ya pueden utilizar y dirigirse a las instituciones europeas en otras lenguas cooficiales. En el caso de España, por ejemplo, el Consejo de la UE llegó a un acuerdo para permitir el uso del gallego, del euskera y del catalán/valenciano mediante la firma de convenios administrativos entre el Gobierno y las diferentes instituciones comunitarias. En la actualidad, los ciudadanos pueden dirigirse a distintos organismos públicos, tales como la Comisión Europea, la Eurocámara, el Consejo de la UE, el Comité de las Regiones y el Defensor del Pueblo de la UE en una de estas lenguas, y recibir contestación igualmente en ellas. Asimismo, no solo los textos jurídicos de los acuerdos comunitarios se traducen a estas lenguas cooficiales sino que también los representantes de las comunidades autónomas afectadas que participan en los Consejos de ministros y en el Comité de las Regiones pueden, previa notificación, intervenir en estas lenguas.

Tal diversidad de lenguas dentro del contexto de las instituciones europeas implica elevados costes de traducción. En un comunicado del 20 de diciembre de 2006, Bruselas indicó que los costes de traducción para toda la UE en 2006 se elevaron a ochocientos millones de euros. Por lo que respecta a la interpretación, el gasto total en la UE en 2005 alcanzó la cifra de ciento noventa millones euros, lo que corresponde a 0,42 euros por ciudadano y año.

Estos datos confirman el importante mercado de los idiomas no solo en la Unión Europea, sino también en cada uno de los Estados miembros donde se fomenta la enseñanza/ aprendizaje de cuantas más lenguas posibles para "facilitar la movilidad, profesional y personal, dentro del mercado único, y favorecer los contactos entre culturas y la comprensión mutua"2. Asimismo, según indica el Eurobarómetro de mayo-junio 2005, publicado en septiembre del mismo año, la mitad de los ciudadanos de la Unión Europea afirma que puede mantener una conversación por lo menos en un idioma además de su lengua materna. Sin embargo, los porcentajes difieren según los países y los grupos sociales: mientras el 99 por ciento de los luxemburgueses conocen como mínimo una lengua además de su lengua materna, el 64 por ciento de los españoles reconoce que solo domina su lengua materna. Si nos referimos a la lengua inglesa, la más estudiada en el conjunto de los países europeos, el 43 por ciento de los ciudadanos de la UE pueden mantener una conversación en inglés frente al 18 por ciento de los españoles ${ }^{3}$.

\section{El caso de España}

En España, la importancia de los idiomas puede variar significativamente según la comunidad autónoma, si bien es cierto que el idioma que centra el mayor interés es el inglés, idioma que se imparte desde edades muy tempranas. Ya sea porque se trata de una lengua con raíces más germánicas que latinas, porque hasta hace unas décadas la orientación preferente era hacia el francés o porque el dominio del inglés es una asignatura pendiente, en estos momentos, 
España está intentando seguir la tendencia según la cual el aprendizaje de un idioma es una cuestión a largo plazo. Por esa razón la totalidad de centros de enseñanza primaria imparten inglés en edades cada vez más tempranas. Los datos que manejan las instituciones europeas -datos cuantitativos y no cualitativos- avalan la conclusión de que en España el estudio del inglés en el sistema público de educación está firmemente establecido.

Tabla 1. Porcentaje de alumnos y lenguas más estudiadas (Curso 2001-2002)

\begin{tabular}{ccccccc}
\hline \multirow{2}{*}{ Lenguas } & \multicolumn{3}{c}{ Educación Primaria } & \multicolumn{4}{c}{ Educación Secundaria } \\
& UE-25 & España & UE-25 & España & UE-25 & España \\
\hline Inglés & 46,4 & 85,2 & 87,4 & 97,5 & 90,5 & 95,9 \\
Alemán & 6,5 & 0,3 & 19,6 & 1,9 & 26,4 & 1,1 \\
Francés & 3,2 & 2,6 & 27,4 & 39,4 & 25,7 & 27,7 \\
Español & $(:)$ & $(-)$ & 7,1 & $(-)$ & 15,0 & $(-)$ \\
\hline
\end{tabular}

Fuente: Eurydice (2005)

Observaciones: (:) datos no disponibles, (-) datos no significativos.

Sin embargo, los esfuerzos del Ministerio de Educación español en cuanto a la imposición del estudio de al menos una lengua extranjera en la escuela no parece ser indicador de la competencia lingüística de los españoles al ver el porcentaje de los que afirman poder mantener una conversación en inglés, por ejemplo. Esta triste realidad ha sido demostrada y puesta en evidencia por muchos estudios empíricos e investigaciones llevadas a cabo en distintas comunidades como Galicia (Vez y Martínez Piñeiro 2002) o Canarias (Wood et al. 2005 y 2007).

La voluntad del Consejo de Europa, a través de la División de Políticas Lingüísticas, de promover la diversidad lingüística y cultural también se ha hecho patente en la enseñanza superior. Hoy en día, un dominio avanzado de idiomas, principalmente del inglés, se ha convertido en requisito casi imprescindible para cursar estudios superiores. Al sentar las bases para la convergencia europea en materia de formación universitaria, la Declaración de Bolonia (1999) da mucha importancia a la libre circulación de estudiantes y profesores entre universidades, por lo que ya no basta con un conocimiento superficial del idioma, sino que se requieren habilidades lingüísticas avanzadas de lectura, comprensión y expresión tanto oral como escrita. Por esta razón, ya desde 1987 la Unión Europea creó el Programa Erasmus para mejorar la calidad y fortalecer la dimensión europea de la enseñanza superior fomentando la cooperación transnacional entre universidades, estimulando la movilidad en Europa y mejorando la transparencia y el pleno reconocimiento académico de los estudios y cualificaciones en toda la Unión. Como bien es sabido, el inglés se ha erigido en lengua común para la comunidad científica universitaria y todo apunta a que continuará siéndolo en los próximos años. Asimismo, también es cierto que la movilidad y los intercambios con múltiples áreas geográficas crean la necesidad de conocer otras lenguas. 


\section{El contexto de las Facultades de Traducción e Interpretación}

Dentro de este contexto alentador, las Facultades de Traducción españolas han sabido adaptarse gradualmente al espacio europeo y desempeñan en la actualidad un papel esencial en la construcción de la Europa plurilingüe; al ofrecer una adecuada combinación de posibilidades lingüísticas y culturales, estos centros suscitan en el alumno el entusiasmo por los idiomas y los preparan para formar parte de una sociedad abierta a otras culturas, en la cual podrán conocer gentes de países y tradiciones muy diversas. Los estudios de traducción les ayudan a desarrollar todo el abanico de sus posibilidades de comunicación, entre las que se incluye, por supuesto, su lengua materna, y todo ello junto con la dimensión intercultural, para convertirlos en unos auténticos profesionales. Y quien habla de profesionalidad en el mundo de la traducción habla, entre otras cosas, del dominio de varios idiomas.

En España, como en muchos países del entorno europeo, la enseñanza universitaria de la traducción y la interpretación clasifica las lenguas en tres grupos, A, B y C. Se trata de una división relativamente antigua que tiene su origen en la interpretación de conferencias desde una perspectiva de mercado en los países del norte de Europa desde hace cuarenta años. Las definiciones no son exactas y permiten una cierta holgura en su interpretación, pero su uso está muy arraigado y no parece que se vaya a cuestionar en un futuro cercano. Se considera lengua A la lengua materna del estudiante, o la lengua de la que el estudiante tiene el nivel C del Common European Framework of Reference o bien la lengua o lenguas oficiales del territorio en el que se sitúa la institución docente.

Se denomina Lengua B a lengua extranjera que el estudiante debe dominar en el momento de comenzar sus estudios. Este dominio es el que se concreta en el nivel B del Common European Framework of Reference. En el caso de España, hasta el momento las lenguas B han sido el inglés (en todos los centros de traducción), el francés, el alemán (en muchos) y el árabe en la Universidad de Granada. Otra característica de las lenguas B es que en ellas los egresados deben contar con destrezas activas y pasivas, esto es, deben poder traducir o interpretar de esas lenguas hacia la suya pero también de la suya hacia esas lenguas.

Con la denominación de lengua $\mathrm{C}$ hacemos referencia a la segunda lengua extranjera que el alumno elige de acuerdo con las diferentes combinaciones que se le ofrecen en el Plan de Estudios y de la que no ha de poseer necesariamente preparación previa alguna. Es de todos sabido que el traductor debe conocer varios idiomas, algunos de forma activa y otros de forma pasiva. Así lo expresa V. García Yebra (1983: 118):

\footnotetext{
El traductor, más aún que el autor original, necesita un conocimiento muy amplio de las lenguas que maneja para su trabajo: conocimiento, al menos pasivo, de la lengua o lenguas de las que traduce; conocimiento activo -que supone, naturalmente, el conocimiento pasivo- de la lengua a la que traduce.
}

La lengua $\mathrm{C}$ es por tanto una tercera lengua (después de $\mathrm{A}$ y $\mathrm{B}$ ) con la que el alumno inicia un aprendizaje nuevo, a un ritmo rápido, pues se intenta llegar a un mismo grado de dominio en comprensión que con la lengua B, o sea su primera lengua extranjera. El número de horas lectivas 4 para ese nuevo aprendizaje de la tercera lengua -denominada " $C$ "- es de gran ayuda para conseguir los objetivos marcados. Además, todo alumno que cursa una lengua $\mathrm{C}$ conoce ya los hábitos y mecanismos que se necesitan para el aprendizaje de una lengua extranjera pues muy posiblemente ya tiene amplios conocimientos de lo que es su lengua B, en la que además estará profundizando en paralelo con el estudio inicial de su lengua C. 
En teoría y tal como señalamos anteriormente, la lengua $\mathrm{C}$ suele entenderse como lengua de la que solo se espera una competencia pasiva (prueba de ello es que no existe materia de traducción A/C dentro de las asignaturas obligatorias); no obstante, conviene resaltar que al llegar al tercer curso del Grado de Traducción, los niveles de Lengua $\mathrm{C}$ son a menudo casi equiparables a los de Lengua B, por lo menos en los concerniente a la comprensión, lo que es todo un logro, en gran parte propiciado por el gran número de horas lectivas que se han concedido a la citada asignatura.

En el caso de España, las lenguas C que se suelen ofrecer en el Grado de Traducción son: el inglés, el francés, el alemán, el italiano y el portugués. Sin embargo, existe un segundo grupo de lenguas C, cuya implantación en la docencia secundaria es mínima o inexistente; entre otras, se encuentran el árabe, el chino, el danés, el griego, el japonés, el neerlandés y el polaco.

Finalmente, a las categorías de lenguas A, B y C se han sumado las lenguas D para responder, en cierta manera, a la creciente demanda de algunas lenguas minoritarias o, simplemente, para hacer frente a la diversidad lingüística de la Unión Europea y ampliar las posibilidades de combinaciones dentro de los estudios de traducción de cada universidad. En cuanto a las lenguas D, cabe destacar algunos aspectos: en primer lugar, que su oferta no es muy amplia y que esta depende en gran medida de la disponibilidad de profesorado y en algunos casos de la situación geográfica del centro universitario. En segundo lugar, que en las Facultades solamente se imparte lengua y cultura, pero ninguna materia relacionada con su traducción o interpretación. En último lugar, son muchos los alumnos que en sus intercambios Erasmus cursan otras lenguas que no se imparten en su Facultad y estas se incluyen en su expediente como Lenguas D. Finalmente, es preciso señalar que, al contrario de lo que ocurre con las lengua A, B y C, cursar una lengua D no es en ningún caso obligatorio sino un plus para la formación de los futuros traductores e intérpretes.

\section{Reflexiones sobre el papel de la segunda lengua extranjera en los estudios de traducción}

Una vez definido el marco contextual de la enseñanza de lenguas extranjeras en su dimensión tanto europea como española, y para el propósito del presente artículo, resulta oportuno realizar algunas reflexiones sobre el papel de la segunda lengua extranjera en los estudios de traducción en España, principalmente en cuanto al tratamiento del componente cultural en la didáctica de la segunda lengua extranjera, o sea, la lengua $\mathrm{C}$.

Tal como lo definen los descriptores del Boletín Oficial del Estado, el objetivo que se tiene que perseguir en la lengua $\mathrm{C}$ es el de profundizar en el estudio de la lengua orientado a la traducción con especial insistencia en los aspectos contrastivos y comunicativos para que, al finalizar su tercer curso del grado de traducción e interpretación -último curso en el que se imparten asignaturas de lengua, siendo el cuarto curso un año de especialización- el alumno tenga un dominio "pasivo", esto es, de recepción de la lengua C, equiparable al de la lengua B. Además, como profesores de la asignatura de Lengua C-Inglés, hemos definido, entre nuestros objetivos principales para el tercer curso los siguientes: profundizar en las funciones textuales y en las referencias culturales; y desarrollar la capacidad de hacer un análisis cultural e intercultural de las lenguas de trabajo del alumno para lograr una traducción 
exacta en contenido e intención. Igualmente, aunque de manera menos intensiva, en los cursos de primero y segundo, los objetivos hacen referencia a la ampliación del bagaje cultural que la Lengua $\mathrm{C}$ proporciona, así como a la conciencia de que la lengua es reflejo de otra percepción o conocimiento del mundo.

La noción de lengua y cultura como binomio indisociable en el proceso traductológico ha sido destacado por numerosos teóricos de la traducción, entre los que cabe resaltar a Nida (1964), por ser uno de los primeros que ha puesto de relieve el carácter transcultural de la traducción. La lengua es el vehículo mediante el cual nos expresamos y por el que pertenecemos a un determinado ámbito, a una determinada cultura que nos hace ver y concebir el mundo de una manera particular y no de otra. A la hora de traducir hemos de tener presente que esa visión, conocimiento o percepción del mundo que refleja la lengua desde la que traducimos es el conjunto de una compleja y dinámica serie de ideas interrelacionadas y, a menudo, contradictorias, de observaciones, de símbolos y prácticas que en su totalidad definen la cultura del autor que traducimos y que hemos de transferir a nuestra lengua/cultura.

La cultura, por tanto, se ve necesariamente reflejada a través de la lengua. Según afirma Martínez, el traductor, que ha de ser un mediador intercultural, que traspasa imágenes y palabras de una lengua/cultura a otra, se mueve dentro de la cultura, ya que ésta engloba todo: "Traducir es un acto, además de interlingüístico, intersemiótico o intercultural." (Martínez 1996: 183) Por tanto, el hecho de trasmitir cultura conlleva una gran responsabilidad y exige del traductor una buena formación en este sentido.

Aunque seamos muy conscientes del nivel medio que generalmente tienen los estudiantes de nuestras titulaciones en sus segundas lenguas extranjeras, creemos que los que nos dedicamos a la Lengua C-Inglés ya podemos contar con una base lingüística suficiente de lengua "pasiva" para poder hacer hincapié en los componentes culturales, los cuales permiten una comprensión profunda de la lengua, conditio sine qua non para la correcta traducción (Luque y Manjón 2003). En efecto, nuestros futuros traductores han de adquirir una competencia que vaya más allá de los conocimientos que tanto gramáticas como diccionarios pueden ofrecer. El estudio de la lengua extranjera para traductores debería centrarse especialmente en el análisis de aquellos aspectos de la lengua relevantes para la traducción. Estos aspectos se encuentran en gran parte en el ámbito de los componentes culturales. Abordar el análisis de la lengua extranjera desde una perspectiva cultural puede facilitar al futuro traductor la comprensión y el dominio de la misma.

Cómo hacer llegar al alumno de traducción e interpretación las herramientas necesarias para poder afrontar esta tarea con éxito es el propósito de los párrafos que vienen a continuación.

Si lo miramos desde el punto de vista de la práctica profesional, no puede haber duda sobre si la traducción desde y a una segunda lengua tiene sentido o no lo tiene. En el mundo de la traducción escrita, no existe separación entre lengua B y lengua C. El profesional que se dedica a la traducción puede verse fácilmente en la situación de tener un encargo, independientemente de que se trate de su primera o su segunda lengua extranjera. El cliente no suele preocuparse de la división que en el ámbito universitario existe entre las dos lenguas extranjeras y el nivel de calidad que exige es el mismo para cualquiera de ellas. Por lo tanto, como docentes de lengua $\mathrm{C}$, hemos de preparar lo mejor posible a nuestro alumnado para este reto. 
A grandes rasgos, las clases de lenguas extranjeras para traductores se han ocupado, por un lado, de la estructura gramatical de la lengua y, por otro, de desarrollar las cuatro habilidades comunicativas (comprensión y expresión oral y escrita), si bien es cierto que se tiende a priorizar cada vez más el trabajo con textos, incluso documentos semi-especializados y especializados.

En nuestra opinión, el estudio de la lengua también debería dejar paso al estudio de esos aspectos tan relevantes para la traducción como, hemos apuntado, son los componente socioculturales, o sea, aquellos elementos que plantean problemas al alumno que no puede solucionar ni con una gramática ni con un simple diccionario, pero que le obligan a reflexionar sobre la idea de que la cultura forma parte integrante de la lengua, y viceversa. Como ya hemos señalado, el nuevo escenario de la Unión Europea, e incluso el más amplio de la globalización de los intercambios económicos a nivel mundial, está teniendo repercusiones en el modo de abordar la enseñanza de las lenguas, orientando esta última hacia la dimensión intercultural. Las mismas directrices europeas ${ }^{5}$ y las establecidas por las administraciones educativas en los currículos de lenguas extranjeras de los Estados miembros de la Unión están empezando a reflejar claramente los más recientes desarrollos en el campo de la didáctica de las lenguas en cuanto a la integración de lengua y cultura en la enseñanza (Buttjes y Byram 1991; Byram 1997, Byram y Risager 1999; Byram y Fleming 2001; Kramsch 1993; Zarate 1986 y 2002), y a señalar como objetivos finales los de ampliar el conocimiento de los alumnos profundizando en la comprensión de las poblaciones que hablan las lenguas que están estudiando, de sus costumbres y modos de vida.

Todos estos aspectos cobran mucha importancia en los estudios de traducción donde los profesores de lengua extranjera en general, y los de lengua $\mathrm{C}$ en particular, deberían centrar el aprendizaje de los futuros profesionales en cuatro aspectos fundamentales relacionados con la competencia sociocultural:

1. La reflexión sobre la idea de que lengua y cultura son realidades indisociables en la traducción.

2. La formación del futuro traductor como individuo competente en diversas lenguas y en sus respectivas culturas (competencia plurilingüe y pluricultural).

3. La formación del futuro traductor como agente social y mediador intercultural y pragmático capaz de integrar y actualizar sus conocimientos en su segunda lengua para poder desarrollar sus competencias en diversas situaciones y contextos como persona que transita de una cultura a otra, creando de este modo espacios de diálogo intercultural.

4. La adquisición de una visión crítica en el plano cultural, siendo consciente de los posibles conflictos entre culturas y sabiendo identificar e interpretar dichos conflictos.

Estos aspectos harán que nuestros alumnos se conciencien de que el modelo del hablante nativo no es válido cuando la finalidad que se persigue es la de desarrollar una competencia plurilingüe y pluricultural. Su meta principal será adquirir las destrezas y habilidades interculturales que les permitan convertirse en personas mediadoras capaces de introducirse en un contexto de identidades múltiples. Como señala María Silvina Paricio, dichas destrezas y habilidades remiten a cuatro capacidades:

Las destrezas y habilidades interculturales remiten a cuatro capacidades: la capacidad de establecer relaciones entre la cultura de origen y la extranjera; la sensibilidad cultural y la capacidad de emplear estrategias variadas para establecer contacto con personas de otras culturas; la capacidad de desempeñar el papel de intermediario cultural entre la propia cultura y la extranjera y de resolver situaciones de conflicto y malentendidos culturales; y la capacidad para superar estereotipos (Paricio 2004: 6). 
A la vista de los planteamientos anteriores, cabe preguntarse cuál debería ser el papel del profesor de lengua $\mathrm{C}$ en una Facultad de Traducción e Interpretación a la hora de desarrollar esta dimensión intercultural en el aula. El docente, también como mediador entre culturas, debe ir mucho más allá de la mera transmisión de información sobre aspectos culturales de un país determinado y enfocar las competencias hacia la acción, o sea, desarrollar en el alumno capacidades que le permitirán, en el futuro, tener una perspectiva cultural crítica y trabajar de manera autónoma sobre el material que va a traducir. Por lo tanto, enfocamos nuestra docencia hacia procedimientos como la observación, la identificación y la comparación de la cultura extranjera con la cultura propia. En este punto, creemos que el descriptor que el Ministerio de Educación propone en el BOE (Boletín Oficial del Estado no 225 de 19-9-2000) para la Lengua C-1 Inglés -"Estudio de la Lengua C orientado a la traducción con especial insistencia en los aspectos contrastivos y comunicativos"- resulta más bien incompleto al no hacer hincapié en lo que consideramos esencial cual es el aspecto sociocultural. A título de sugerencia, incluimos a continuación algunas indicaciones prácticas que favorecerían este aprendizaje en las clases de lengua $\mathrm{C}$ para traductores:

1. Dar importancia a la ejemplificación de los elementos no-lingüísticos. Un primer acercamiento a la importante relación lengua/cultura se podría hacer con la presentación de este diálogo entre una niña y su padre que simboliza la relación entre la obsesión por enseñar aspectos meramente lingüísticos y el fracaso en los objetivos de aprendizaje consecuentes:

\footnotetext{
"Papá, ¿Por qué los franceses agitan tanto los brazos?”. “A qué te refieres?”. "Me refiero a que cuando hablan mueven los brazos sin parar". Después de hablar un rato con su padre, quien no le ofrece ningún tipo de explicación de esta peculiaridad de los franceses, pero que toma en serio sus intentos de explicar la causa de este comportamiento, la niña dice finalmente: "Papá, cuando nos enseñan francés en el colegio, ¿por qué no nos enseñan a mover los brazos?". Y su padre le contesta: "No lo sé. De verdad no lo sé. Probablemente por eso a la gente le cuesta tanto aprender idiomas. De todos modos, es absurdo: la idea de que el idioma está compuesto de palabras es completamente ridícula” (Fleming 1998: 183).
}

Gracias a este corto diálogo, se le pedirá al alumno de lengua C que reflexione sobre lo que se debería integrar en la práctica docente, sobre la importancia de la dimensión sociocultural y sobre la perspectiva intercultural en los estudios de traducción. En efecto, la cultura meta siempre va anexa a la lengua que se aprende.

2. Contrastar la cultura meta y la propia, reconocer las similitudes y diferencias, entender y valorar las culturas, ya que siempre se acerca uno a la cultura extranjera y a los valores de aquella a través de su propia cultura y valores. Cuantas más lenguas y culturas conozca el alumno, más receptivo será ante la alteridad y más curioso y abierto hacia otros modos de entender la realidad de diversos países.

3. No caer en tópicos ni estereotipos, y profundizar en una reflexión crítica.

4. Seguir trabajando la lengua desde la perspectiva cultural. No se trata de impartir una clase de cultura y civilización olvidándose del elemento lingüístico, sino de poner en práctica los elementos estudiados y aprendidos a través de trabajos de producción tanto escrita como oral a partir de situaciones reales de comunicación, incluso de traducción. Para aprender los modelos culturales de los hablantes de la segunda lengua extranjera, nuestros alumnos han de verse en situaciones lo más verosímiles posible que les permitan acercarse, por ejemplo, a formas coloquiales, modismos, expresiones propias de grupos sociales, profesionales, argot, etc. 
5. Emplear material adecuado donde los contenidos socioculturales no están aislados o presentados en un apartado especial. Trabajar con una gama lo más amplia posible de materiales auténticos para identificarse con las perspectivas y experiencias de las personas que viven en los países donde se habla la lengua objeto de estudio: textos, grabaciones, cartas, fotografías, dibujos, películas, etc.

6. Investigar e informarse sobre aspectos de los países donde se habla la lengua estudiada. Los mismos alumnos pueden implicarse en la búsqueda de documentos que se vayan a utilizar en clase. Desde el punto de vista de la traducción, han de buscar el contexto en el que este documento ha sido producido y la intención que tienen.

7. Fomentar el trabajo en grupo, el diálogo, el debate y la participación activa y reflexiva.

8. Fomentar los contactos en el aula o fuera de ella con hablantes nativos de la Lengua $\mathrm{C}$ que se estudia para que a través de la comunicación directa y real se puedan apreciar los diversos componentes de la lengua, desde el lenguaje corporal, pasando por las estructuras lingüísticas hasta las expresiones y formas culturales.

La última reflexión que quisiéramos proporcionar tiene que ver con el nivel de especificidad de las lenguas para traductores. Aunque en España continúe el debate en torno a la calificación de las lenguas extranjeras para traductores como lenguas para fines específicos, nosotros planteamos el hecho de que el aprendizaje de lenguas en las Facultades de Traducción e Interpretación tiene, sin lugar a dudas, finalidades prácticas incontestables, por lo que la presencia del componente cultural se tiene que examinar desde dos perspectivas bien definidas: una relacionada con los contenidos temáticos de una disciplina determinada (por ejemplo, el lenguaje jurídico), y otra que depende del uso de la lengua como instrumento de comunicación entre los miembros de un grupo. Asimismo, los médicos o juristas de diferentes nacionalidades constituyen un grupo cultural más homogéneo que un negociante de Londres y otro de Sydney, que tienen profesiones menos vinculadas a una materia determinada y cuyas relaciones pueden tener un fuerte peso cultural que depende de una serie de normativas y costumbres aceptadas en una comunidad y no en la otra. En el segundo caso, el acercamiento a la cultura es exactamente el mismo que el que explicamos anteriormente, pero en el primer caso, se trata de una cultura técnica específica que el alumno deberá adquirir a lo largo de sus estudios y luego en su vida profesional. Ahora bien, insistimos en que la falta de conocimientos en la lengua general irá en detrimento de su competencia intercultural. Cualquiera que sea su forma, oral o escrita, la lengua siempre comprende momentos de intercambio en los que se discurre sobre temas más generales.

\section{Conclusión}

Teniendo en cuenta todo lo mencionado, se puede afirmar que el enfoque cultural en la clase de segunda lengua para traductores sugiere que el alumno tome conciencia de los hábitos arraigados en las relaciones humanas cotidianas y en el mundo profesional, así como sus repercusiones lingüísticas para adquirir gradualmente una competencia intercultural que le permita desenvolverse en su futura labor como traductor. Sin lugar a dudas, la competencia intercultural plantea un reto para todos los profesores de idiomas y aún más para los profesores de segundas lenguas extranjeras que, cabe recordarlo, suelen trabajar con un alumnado con competencias lingüísticas y culturales menos desarrolladas. 


\section{Notas}

1. Esta investigación hace parte del proyecto "La interculturalidad en la enseñanza de lenguas extranjeras" dentro del grupo de investigación "La adquisición de lenguas/culturas extranjeras: procesos cognitivos y competencia estratégica" de la Universidad de Las Palmas de Gran Canaria.

2. http://europea.eu/languages/es/chapter/14.

3. http://www.noticiascadadia.com/noticias/articulo/el-43-de-los-ciudadanos-de-la-ue-pueden-manteneruna-conversacion-en-ingles-frente-al-18-de-los.htm.

4. Seis horas semanales en el primer curso, seis en el segundo y cuatro en el tercero.

5. El Marco de referencia europeo para el aprendizaje, la enseñanza, y la evaluación de las lenguas concede un papel importante a la dimensión intercultural.

\section{Bibliografía}

Boletín Oficial del Estado. No. 225 de 19 de septiembre de 2000. (Resolución de 16 de agosto de 2000).

Buttjes, D. y M. Byram. 1991. “Introduction”. En: D. Buttjes y M. Byram (eds.), 1- 12.

Buttjes, D. y M. Byram (eds.). 1991. Mediating Languages and Cultures: Towards an Intercultural Theory of Foreign Language Education. Clevedon, Multilingual Matters.

Byram, M. 1997. Teaching and Assessing Intercultural Communicative Competence. Clevedon, Multilingual Matters.

Byram, M. y M. Fleming. 2001. Perspectivas interculturales en el aprendizaje de idiomas. Enfoques a través del teatro y la etnografía. Madrid: Cambridge University Press.

Byram, M. y K. Risager. 1999. Language Teachers, Politics and Cultures. Clevedon, Multilingual Matters.

Comisión Europea. 1999. Unión Europea. Recopilación de los Tratados. Tomo I, Vol. I, Luxemburgo: Oficina de publicaciones oficiales de las Comunidades Europeas.

Eurobarómetro. 2007. "El 43\% de los ciudadanos de la UE pueden mantener una conversación en inglés frente al 18\% de los españoles que manifiestan saber hablar en inglés". Noticiascadadía. 17 de abril. http://www.noticiascadadia.com/noticias/articulo/el-43de-los-ciudadanos-de-la-ue-pueden-mantener-una-conversacion-en-ingles-frente-al-18de-los.html. Consulta: 4 de octubre de 2007. 
Eurydice. 2005. Key data on teaching languages at school in Europe. http://www.eurydice. org/Documents/KDLANG/2005/EN/FrameSet.htm. Consulta: 3 de octubre de 2007.

Fleming, M. 1998. Sensibilidad cultural y formas de teatro. Perspectivas interculturales en el aprendizaje de idiomas. Londres: Cambridge University Press.

García Yebra, V. 1983. En torno a la traducción. Madrid: Gredos.

Kramsch, C. 1993. Context and Culture in Language Teaching. Oxford: Oxford University Press.

Luque Durán, J. y F. Manjón Pozas. 2003. “Claves culturales del diseño de las lenguas: Fundamentos de tipología fraseológica”. Elies. 16. http://elies.rediris.es/elies16/Claves. html. Consulta: 15 de septiembre de 2007.

Martínez García, A. 1996. “Cultura y traducción”. Contrastes. Revista Interdisciplinar de Filosofía. Universidad de Málaga. 1: 173- 190.

Nida, E. A. 1964. Toward a Science of Translating, with special reference to principles and procedures involved in Bible translating. Leiden, E. J. Brill.

Paricio, M. S. 2004. "Dimensión intercultural en la enseñanza de las lenguas y formación del profesorado". Revista Iberoamericana de Educación, Organización de Estados Iberoamericanos. 34 (4). http://www.rieoei.org/deloslectores/810Paricio.PDF. Consulta: 30 de septiembre de 2007.

Portal de la Unión Europea. http://europa.eu/languages/es/chapter/14. Consulta: 28 de septiembre de 2007.

Vez, J. M. y M. Martínez Piñeiro (eds.). 2002. Competencia comunicativa oral en lenguas extranjeras: Investigación sobre logros del alumnado gallego de inglés y francés al finalizar la ESO. Santiago de Compostela: ICE-Servicio de Publicaciones de la USC.

Wood, M., M. Peñate y P. Bazo (eds.). 2004. FreconWin: corpus canario de inglés escrito. Las Palmas de Gran Canaria: ICEC.

2007. FreconWin: corpus canario de inglés oral. Las Palmas de Gran Canaria: ICEC.

Zarate, G. 1986. Enseigner une culture étrangère. París: Hachette.

2002. Las competencias interculturales: del modelo teórico al diseño curricular. Madrid, Universidad Antonio de Nebrija, Aula de Español. 